\title{
Improvement of electrospun polymer fiber meshes pore size by femtosecond laser irradiation
}

\author{
Esther Rebollar ${ }^{\mathrm{a}, *}$, Diego Cordero ${ }^{\mathrm{a}}$, Albino Martins ${ }^{\mathrm{b}}$, Stefano Chiussi ${ }^{\mathrm{a}}$, Rui L. Reis ${ }^{\mathrm{b}}$, \\ Nuno M. Neves ${ }^{b}$, Betty León ${ }^{\mathrm{a}}$ \\ a Departamento de Física Aplicada, E.T.S.I. Industriales, Universidad de Vigo, Rúa Maxwell s/n, Campus Lagoas-Marcosende, 36310 Vigo, Spain \\ b 3B's Research Group-Biomaterials, Biodegradables and Biomimetics, University of Minho, Headquarters of the European Institute of \\ Excellence on Tissue Engineering and Regenerative Medicine, AvePark, 4806-909 Taipas, Guimarães, Portugal
}

\section{A R T I C L E I N F O}

\section{Article history:}

Received 4 October 2010

Received in revised form 2 December 2010

Accepted 2 December 2010

Available online 9 December 2010

\section{Keywords:}

Pore size

Femtosecond laser

Fiber mesh

Electrospinning

Laser ablation

\begin{abstract}
A B S T R A C T
Polymer meshes have recently attracted great attention due to their great variety of applications in fields such as tissue engineering and drug delivery. Poly ( $\varepsilon$-caprolactone) nanofibers were prepared by electrospinning giving rise to porous meshes. However, for some applications in tissue engineering where, for instance, cell migration into the inner regions of the mesh is aimed, the pore size obtained by conventional techniques is too narrow. To improve the pore size, laser irradiation with femtosecond pulses (i.e., negligible heat diffusion into the polymer material and confined excitation energy) is performed. A detailed study of the influence of the pulse energy, pulse length, and number of pulses on the topography of electrospun fiber meshes has been carried out, and the irradiated areas have been studied by scanning electron microscopy, contact angle measurements and spectroscopic techniques. The results show that using the optimal laser parameters, micropores are formed and the nature of the fibers is preserved.
\end{abstract} (c) 2010 Elsevier B.V. All rights reserved.

\section{Introduction}

Polymer meshes have received significant attention in the last few years as strongly contributing to the development of advanced materials for a great variety of applications such as tissue engineering (membranes for skin, tubes for blood vessels, 3D scaffolds for bone and cartilage regeneration, and implant interfaces) [1-4], wound dressing [5], and drug delivery [6]. Polymer fiber meshes also have potential use in commodities, and they have become a research target for clothes [7], hygiene applications [8], and filtration, due to their high filtration efficiency and low air resistance [9]. Additionally they can be used in industrial applications, for micro/nano-electronic devices, electrostatic dissipation, electromagnetic interference shielding, photovoltaic devices, LCD devices, high-efficiency catalyst carriers, electrodes for batteries or electrochemical supercapacitor applications [9,10], and for nanosensors taking advantage of their high surface area and good transport properties (thermal, piezoelectric, biochemical, and optical sensors) [11-14].

\footnotetext{
* Corresponding author. Present address: Instituto de Química Física Rocasolano, CSIC, Serrano 119, 28006 Madrid, Spain. Tel.: +34 915619400; fax: +34 915642431. E-mail address: e.rebollar@iqfr.csic.es (E. Rebollar).
}

There are several methods for producing nanofibers, from high-volume production methods such as melt fibrillation [15], island-in-sea [16], and gas jet techniques [17], to highly precise methods like nanolithography, self-assembly and phase separation [18-20]. Electrospinning presents several advantages with its comparative low cost and relatively high production rate. The morphology, fiber diameter and porosity of electrospun nanofibrous scaffolds can be controlled by varying parameters, such as applied electric field strength, spinneret diameter, distance between the spinneret and the collecting substrate, temperature, feeding rate, humidity, air speed, and properties of the solution or melt, such as the type of polymer, its molecular weight and concentration, surface tension, conductivity, viscosity, and temperature [21]. Many polymers, both synthetic and natural, have been successfully electrospun into nanofibers, such as poly(ethylene terephthalate) (PET), polystyrene (PS), poly(vinyl chloride) (PVC), and poly( $\varepsilon$-caprolactone) (PCL), among others [9].

Despite the high level of porosity and high specific surface area of the fiber meshes, the pore size is usually too narrow for some applications in tissue engineering, where cell migration into the inner regions of the fiber-mesh scaffolds is aimed [22]. In fact, most biological studies with electrospun nanofiber meshes show that cells tend to stay at the surface of the meshes. This is a serious limitation which may compromise its use in the regeneration of tissues. Strategies already suggested in literature include the use of 
porogen agents such as salt particles or chemical blowing agents $[23,24]$. However, major research effort is still needed to improve the pore size of electrospun nanofiber meshes. Therefore, in order to address this issue and drill holes without affecting the nature of the polymer fibers, laser processing is being attempted.

Laser processing of materials offers advantages over both chemical and other physical methods. It has proven to be an excellent technique for patterning engineering materials [25], via a singlestep process directly removing the material through ablation. In particular, femtosecond (fs) lasers have gained great interest recently due to the short time scale of laser-material interaction. In femtosecond laser ablation, heat diffusion into the polymer material is negligible, the energy loss into the sample is minimized, and excitation energy is very confined [26]. This aspect makes the use of femtosecond laser pulses very appealing for micropatterning biodegradable polymers [27].

In this work we propose the laser processing of electrospun PCL nanofiber meshes. PCL is a semi-crystalline polyester regarded as a soft and hard-tissue compatible material that has been recently proposed for bone graft substitutes and tissue engineering due to its non-toxic nature and biodegradability. Despite its hydrophobic character being undesirable for in vitro cell culture [28,29], some chemical surface modifications, namely by plasma treatment, significantly improve the cytocompatibility of those electrospun nanofiber meshes [30]. In the literature some reports on laser processed PCL were published recently. PCL thin films were drilled with fs laser, forming precisely defined micro-hole arrays and improving the hydrophilicity and providing enhanced conditions for tissue cells to anchor [31]. Electrospun PCL meshes were also structured by ablation of linear grooves with a scanned femtosecond laser [32]. However, some melting of fibers, which is an undesirable effect, was observed at the edges of grooves.

The aim of this work is to process PCL fiber meshes improving the pore size of the electrospun meshes, which will allow cell migration into the inner regions, without side effects as melting or chemical modifications. To reach that purpose it is important to optimize the laser parameters, and a detailed study of the influence of the pulse energy, pulse length, and number of pulses on the topography of electrospun fiber meshes is reported here.

\section{Experimental}

\subsection{Fiber mesh preparation}

Fiber meshes were prepared by electrospinning as described in detail elsewhere [30]. A polymeric solution of $17 \%(\mathrm{w} / \mathrm{v})$ PCL (TONE $^{\mathrm{TM}}$, Union Carbide Chemicals and Plastics Division; New Jersey) was prepared in a mixture ( $7: 3$ ratio) of chloroform and N,Ndimethylformamide (both purchased from Aldrich). The solution was electrospun at $9-10 \mathrm{kV}$, a needle-to-ground collector distance of $20 \mathrm{~cm}$, and a flow rate of $1 \mathrm{~mL} / \mathrm{h}$. The nonwoven fibrous meshes were collected on a flat aluminium substrate and the solvent evaporation was performed at room temperature during at least 2 days.

The thickness of the fiber meshes was measured by means of an optical microscope (NIKONOPTIPHOT-2) with a magnification of $10 \times$ and a digital indicator Mitutoyo $(\mathrm{S} 1012 \mathrm{M})$ with a resolution of $0.01 \mathrm{~mm}$. The meshes resulted to be $198 \pm 17 \mu \mathrm{m}$ thick.

\subsection{Laser irradiation}

The PCL fiber meshes were laser-processed using a regeneratively amplified Ti:sapphire mode-locked Spectra-Physics Millenia-Pumped Tsunami femtosecond laser in standard room conditions. The femtosecond laser uses Ti:sapphire doped crystal as the medium with wavelength of $800 \mathrm{~nm}$ and pulse duration is varied between 60 and $120 \mathrm{fs}$ for these experiments, at $1 \mathrm{kHz}$ repetition rate.

Surface patterning was performed by using a stage with translation in $x$ and $y$-axis with a resolution of $5 \mu \mathrm{m}$ and the laser beam was focused by a fused silica lens $(f=500 \mathrm{~mm})$.

\subsection{Surface characterization and analysis}

The surface morphology of the laser processed PCL membranes was observed by scanning electron microscopy (SEM), on goldsputtered laser processed meshes using a Philips XL 30 with a resolution of $3.5 \mathrm{~nm}$. Gold sputtering of the fiber meshes was performed in a vacuum chamber with a pressure of $10^{-1} \mathrm{mbar}$ and a current of $25 \mathrm{~mA}$ for a period of $150 \mathrm{~s}$.

The wettability of the surfaces was assessed by contact angle measurements. Measurements of the dynamic contact angle at room temperature were carried out using a pocket goniometer PG2 (FIBRO System) and distilled water as the liquid. Wetting hysteresis was also characterised to determine the highest contact angle as the liquid spreads across the dry surface and the lowest contact angle as the liquid retracts from the wet area on the surface. These two contact angles, referred to as the advancing/receding contact angles describe the wetting hysteresis. Ten measurements were carried out for each sample and statistical analysis was performed.

The chemical modification of the laser processed samples was evaluated by micro FTIR spectroscopy (Thermo Nicolet 6700 coupled to a microscope Continuum) with a resolution of $4 \mathrm{~cm}^{-1}$ and 128 sample scans, and micro-Raman spectroscopy (Jobin Yvon model Labman HR) with excitation wavelength of $488 \mathrm{~nm}$ at a power of $3.7 \mathrm{~mW}$ and a magnification of $50 \times$. The scanning operations and data processing were under the control of a computer and LabSpec software. The resolution is $4 \mathrm{~cm}^{-1}$ and the integration time and number of scans is 15 and 20 s, respectively.

ToF-SIMS measurements were also performed for further characterization of the chemical composition. The mass spectra of the samples were recorded on a ToF-SIMS IV instrument (Ion-Tof $\mathrm{GmbH}$, Germany). The sample was bombarded with a pulsed Bismuth ion beam. The secondary ions generated were extracted with a $10 \mathrm{kV}$ voltage and their time of flight from the sample to the detector was measured in a reflectron mass spectrometer. The typical analysis conditions for this work were $25 \mathrm{keV}$ pulsed $\mathrm{Bi}^{3+}$ beam at $45^{\circ}$ incidence and electron flood gun was used for charge compensation.

\section{Results and discussion}

SEM observation of PCL fiber meshes revealed micro-porous (mean pore size of $2.9 \mu \mathrm{m}$ ), fibrous structures randomly oriented with fiber diameter in the range of some hundred nanometers to a few microns (mean diameter of $540 \mathrm{~nm}$ ) as shown in Fig. 1a.

In a first step, the pulse energy $(E)$ dependence (energies between 100 and $600 \mu \mathrm{J}$ ) was studied. We observed that minimum energies of $100 \mu \mathrm{J}$ are needed to drill holes, as shown in Fig. 1b, and the dimensions of the holes increase with increasing $E$, following the Gaussian distribution of the beam profile, as plotted in Fig. 2. Besides the hole diameter, it is important to observe the morphology of the remaining fibers since the aim is to improve the porosity without any additional effect. Fibers irradiated with one single pulse at energies below $500 \mu \mathrm{J}$ remain apparently unaffected keeping the porosity (Fig. 1c), while in the case of PCL meshes irradiated at higher energies the pores are sealed around the drilled hole (Fig. 1d).

Regarding the effect of pulse duration, this was modified in the range of 60-120 fs. Significant differences were not observed in the 

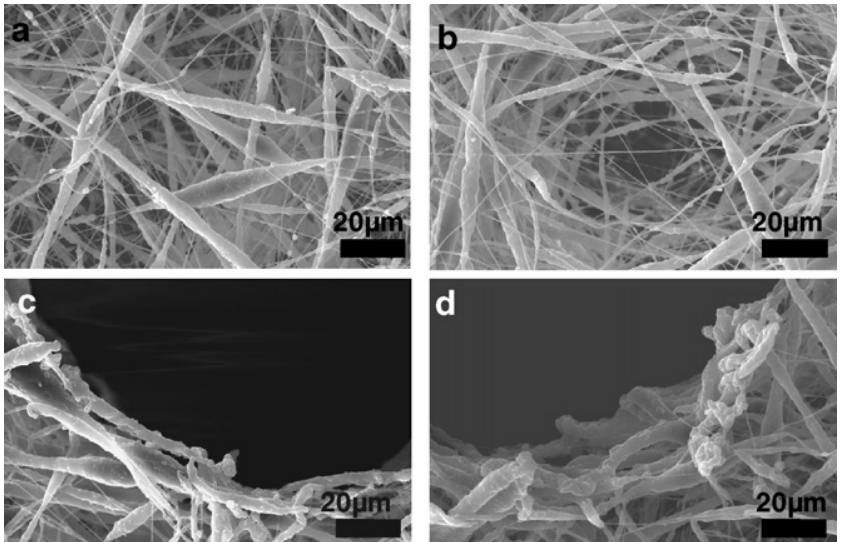

Fig. 1. SEM micrographs of PCL fiber mesh (a) non-irradiated; (b) irradiated with a single pulse of $65 \mathrm{fs}$ and $100 \mu \mathrm{J}$; (c) irradiated with a single pulse of $65 \mathrm{fs}$ and $300 \mu \mathrm{J}$; (d) irradiated with a single pulse of $65 \mathrm{fs}$ and $600 \mu \mathrm{J}$.

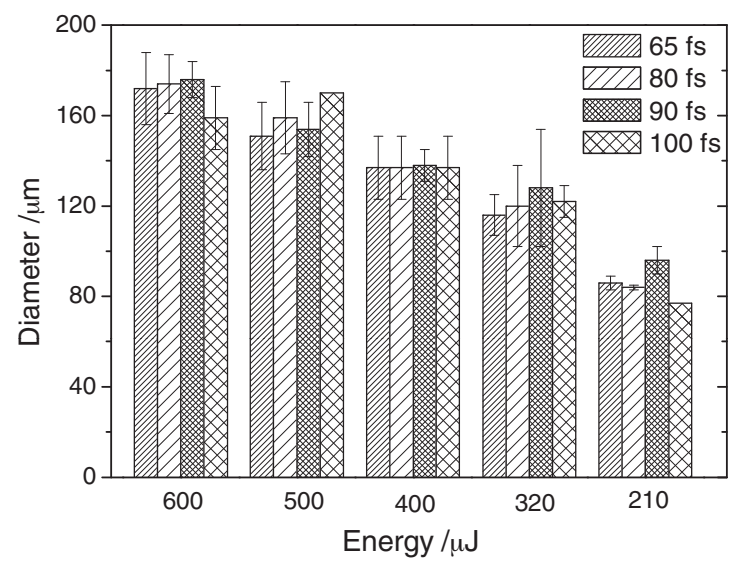

Fig. 2. Dependence of the hole diameter on the laser energy at different pulse duration (each value is the average of 10 different measurements and error bars represent the standard deviation).

size of the holes when one single pulse and the same pulse energy are used (Fig. 2). However, shorter pulses show to be more adequate to avoid additional modification of the fibers, as shown in Fig. 3 which correspond to the holes drilled with 65 and $100 \mathrm{fs}$ pulses, respectively. For this reason pulses of around 65 fs length were used in further experiments.

To study the effect of the number of pulses $N$ on the laser drilling, the PCL fiber meshes were perforated at different energies with $N=1-5$. It can be observed in Fig. 4 that irradiation with two pulses induces the formation of larger holes, but for a higher number of pulses the diameter remains approximately constant. Regarding the morphology of the fibers, the porosity of the mesh is lost around the holes for a number of pulses higher than 2 when the energy is higher than $300 \mu \mathrm{J}$. In Fig. 5, an example is shown, which cor-
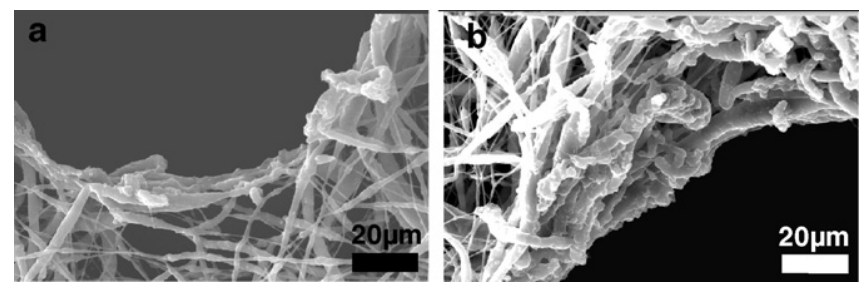

Fig. 3. SEM micrographs of PCL irradiated with a single pulse of $400 \mu \mathrm{J}$ and (a) $65 \mathrm{fs}$; (b) $100 \mathrm{fs}$.

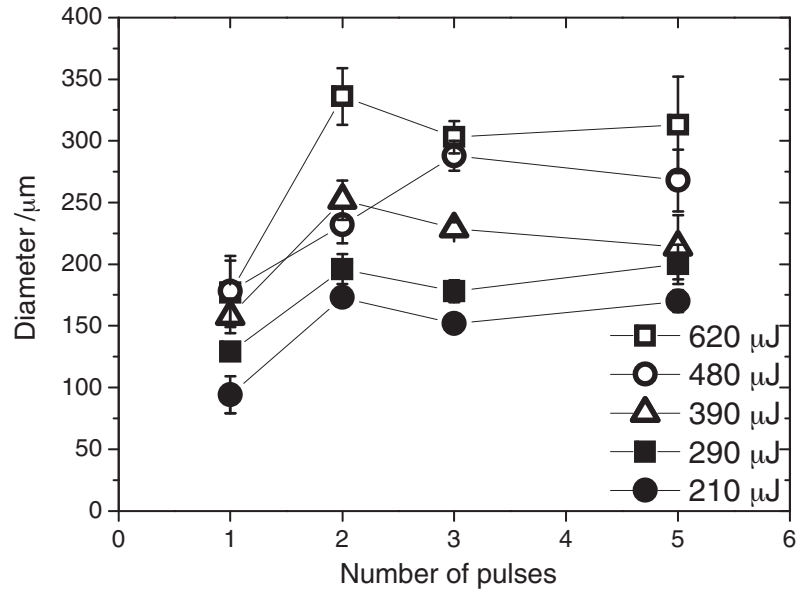

Fig. 4. Dependence of the hole diameter on the number of pulses at different laser energies and pulses of $65 \mathrm{fs}$ (each value is the average of 10 different measurements and error bars represent the standard deviation).

respond to PCL fiber mesh irradiated with 3 pulses of $400 \mu \mathrm{J}$ and $65 \mathrm{fs}$.

The wettability of the surfaces was assessed by contact angle measurements. Samples of $1 \times 1 \mathrm{~cm}^{2}$ were perforated with a matrix of holes produced with one single pulse of $65 \mathrm{fs}$ and $400 \mu \mathrm{J}$. The results previously described showed that pores of $90-200 \mu \mathrm{m}$ can be obtained without additional adverse effects to the structure by optimizing laser energy, laser pulse duration and number of pulses. The selected distance between the centres of the holes was $500 \mu \mathrm{m}$. At time zero the contact angle for non-irradiated and irradiated PCL nanofiber meshes was $129 \pm 3^{\circ}$ and $130 \pm 5^{\circ}$, respectively. These values of the contact angle indicate that the surface of the PCL fiber meshes is hydrophobic. Advancing contact angle is $128 \pm 3^{\circ}$ and $133 \pm 4^{\circ}$, and the receding contact angle is $68 \pm 5^{\circ}$ and $76 \pm 5^{\circ}$ for non-irradiated and irradiated PCL meshes, respectively. Thus, no significant difference regarding the wettability is found between the non-irradiated and irradiated meshes, which could indicate no significant changes in the chemistry of the surfaces induced by laser irradiation.

To discard any possible chemical modification of the PCL nanofiber meshes, non-irradiated and irradiated areas of the same mesh were analyzed by Fourier Transformed Infrared Spectroscopy (FTIR), micro-Raman spectroscopy and Time of Flight-Secondary Ion Mass Spectrometry (ToF-SIMS). FTIR spectra shown in Fig. 6 were recorded from non-irradiated areas and from the edge of the laser drilled holes. Measurements were repeated at five different positions of the sample. The characteristic bands of PCL are observed: symmetrical and antisymmetrical $\mathrm{CH}_{3}$ and $\mathrm{CH}_{2}$ stretching at $2950-2860 \mathrm{~cm}^{-1}, v(\mathrm{C}=\mathrm{O})$ at $1720 \mathrm{~cm}^{-1}, \delta\left(\mathrm{CH}_{2}\right)$ and $\omega\left(\mathrm{CH}_{2}\right)$ at

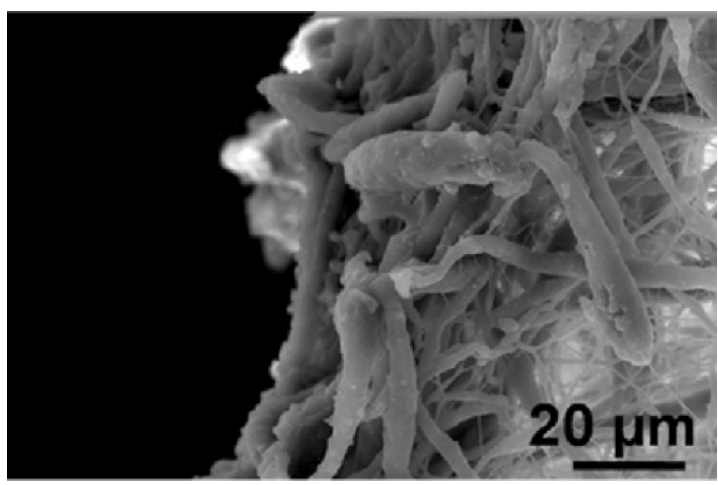

Fig. 5. SEM micrograph of PCL irradiated with 3 pulses of $65 \mathrm{fs}$ and $400 \mu \mathrm{J}$. 


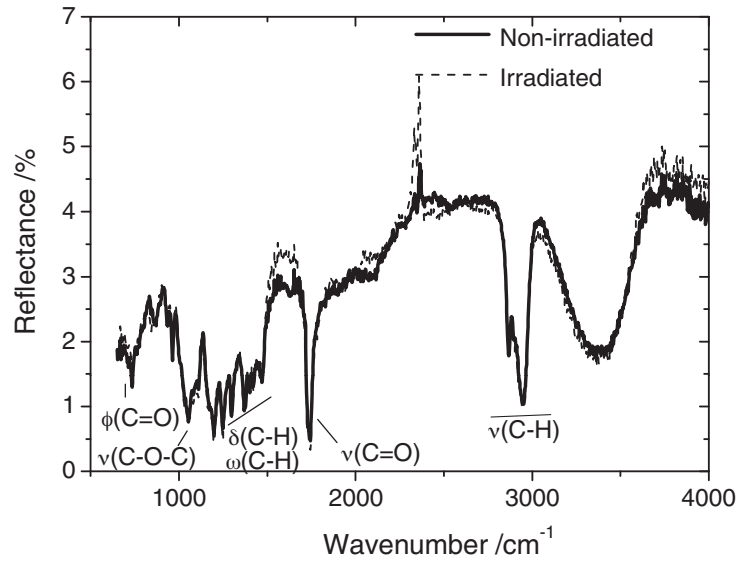

Fig. 6. FTIR spectra of non-irradiated and irradiated ( 1 pulse, $580 \mu \mathrm{J}$, and $65 \mathrm{fs}$ ) PCL fiber meshes.

$1470-1280 \mathrm{~cm}^{-1}, v(\mathrm{C}-\mathrm{O}-\mathrm{C})$ at $1107-1087 \mathrm{~cm}^{-1}$, and $\phi(\mathrm{C}=\mathrm{O})$ rocking at $732 \mathrm{~cm}^{-1}$ [33]. It can be seen that no significant changes are induced by laser irradiation.

Raman spectra were measured for non-irradiated areas and areas at the edge of the holes with a spot size of around $2 \mu \mathrm{m}$. As shown in Fig. 7, the $\mathrm{CH}$ stretching region $\left(2800-3100 \mathrm{~cm}^{-1}\right)$ presents two bands, centered at 2916 and $2866 \mathrm{~cm}^{-1}$. A narrow line is observed at $1720 \mathrm{~cm}^{-1}$ which is assigned to $v(\mathrm{C}=0)$ stretching mode, bands at $1470-1415 \mathrm{~cm}^{-1}$ are assigned to $\delta\left(\mathrm{CH}_{2}\right)$, bands at $1303-1281 \mathrm{~cm}^{-1}$ correspond to $\omega\left(\mathrm{CH}_{2}\right)$, bands at $1107-1033 \mathrm{~cm}^{-1}$ correspond to skeletal stretching and the band at $912 \mathrm{~cm}^{-1}$ is assigned to $v(\mathrm{C}-\mathrm{COO})$ [34]. As in the case of FTIR spectroscopy no significant differences were observed between non-irradiated and irradiated meshes, indicating that no significant chemical modification was induced by laser irradiation.

ToF-SIMS analysis was also performed, as a more sensitive technique than FTIR and Raman spectroscopy. Spectra measured at different positions on the sample are shown in Fig. 8. They represent the distribution of ions by mass-to-charge ratio $(\mathrm{m} / \mathrm{z})$ in a sample. A first spectrum corresponds to an area of $332 \times 332 \mu \mathrm{m}^{2}$ (Fig. 8a), and another spectrum corresponds to a non-irradiated area of $37 \times 37 \mu \mathrm{m}^{2}$, localized at the edge of one hole (Fig. $8 \mathrm{~b}$ ). The recorded spectra are relatively simple and in accordance with spectra reported in the literature [35]. The spectra of irradiated and non-irradiated regions are very similar and the same peaks are detected in both cases, as shown in Fig. 8. The only difference is a decrease in the relative intensity of the peak at $m / z 115$ in comparison with the rest of the peaks, which could be the result of a higher

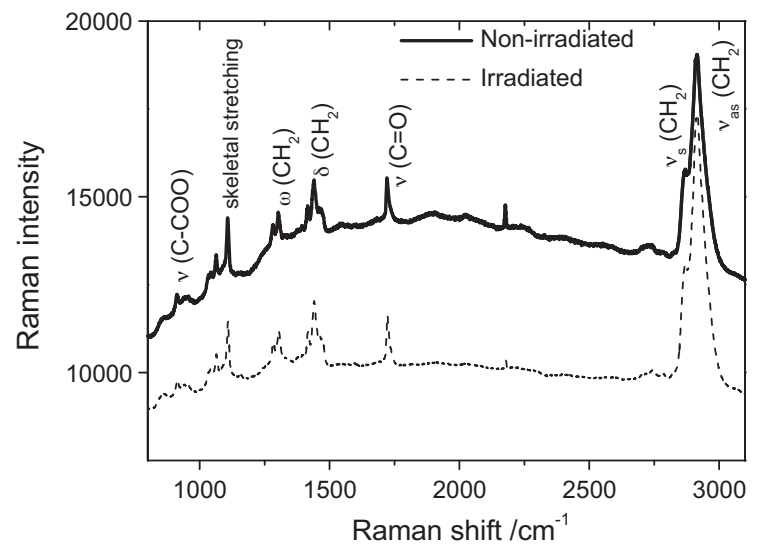

Fig. 7. Micro-Raman spectra of non-irradiated and irradiated ( 1 pulse, $400 \mu \mathrm{J}$, and $65 \mathrm{fs})$ PCL fiber meshes.

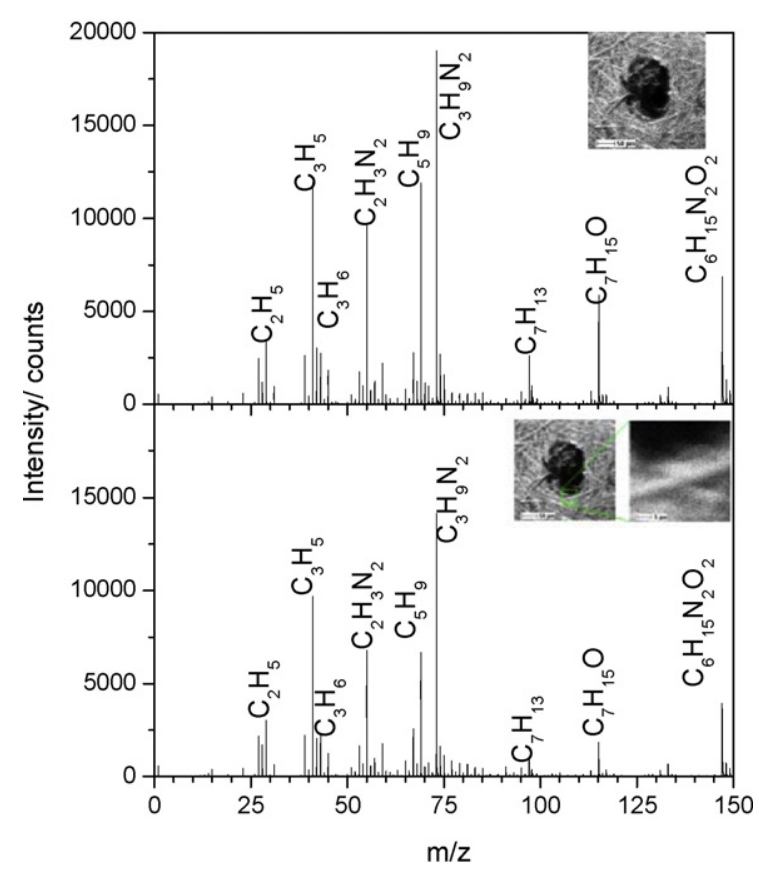

Fig. 8. ToF-SIMS spectra representing the distribution of ions by mass-to-charge ratio at different areas of irradiated PCL meshes with 1 pulse, $580 \mathrm{~mW}, 65 \mathrm{fs}$ : (a) $332 \times 332 \mu \mathrm{m}^{2}$ and (b) $37 \times 37 \mu \mathrm{m}^{2}$ at the edge of the hole.

fragmentation of the molecular ion due to the higher ion bombardment density, since the spectrum is recorded in a much smaller area (one order of magnitude). For further analysis a mapping of the ions was performed and a homogeneous distribution is observed without any preferential location of the different ions. This result indicates that the composition of the fibers is homogeneous.

\section{Conclusions}

The results reported here show that pore size can be controlled by optimizing laser parameters. Additionally, physical and chemical integrity of the electrospun PCL nanofiber meshes is kept, as confirmed by spectroscopic analysis. Therefore, when used properly, femtosecond laser provides an excellent means of micropatterning electrospun polymeric meshes at high level of precision. This paves the way for further enhancement in applications such as membrane tissue engineering.

\section{Acknowledgements}

This work was partially supported by the European Integrated Project GENOSTEM (LSH-STREP-CT-2003-503161), the European Network of Excellence EXPERTISSUES (NMP3-CT-2004-500283), the Interreg III Project Proteus (SP1P151/03), Xunta de Galicia (Consolidación 2006/12) and Xunta de Galicia, Axudas para adquisición de instrumentación científica 2004, PGIDIT04PXI32201IF. ER acknowledges Ángeles Alvariño Program (Xunta de Galicia) and funding from Universidad de Vigo for a stay at 3Bs. AM acknowledges the Portuguese Foundation for Science and Technology for the PhD grant (SFRH/BD/24382/2005).

\section{References}

[1] S.A. Riboldi, M. Sampaolesi, P. Neuenschwander, G. Cossu, S. Mantero, Biomaterials 26 (2005) 4606.

[2] W.J. Li, C.T. Laurencin, E.J. Caterson, R.S. Tuan, F.K. Ko, J. Biomed Mater. Res. 60 (2002) 613.

[3] C.J. Buchko, L.C. Chen, Y. Shen, D.C. Martin, Polymer 40 (1999) 7397. 
[4] L.S. Nair, S. Bhattacharya, C.T. Laurencin, Nanotechnologies and tissue engineering-the scaffold based approach, in: Challa Kumar (Ed.), Nanotechnologies for the Life Science. Vol. 9. Tissue, Cell and Organ Engineering, Wiley-VCH, 2008, pp. 1-65.

[5] S.Y. Silva, L.C. Rueda, M. Lopez, I.D. Velez, C.F. Rueda-Clausen, D.J. Smith, G. Muñoz, H. Mosquera, F.A. Silva, A. Buitrago, H. Diaz, P. Lopez-Jaramillo, Trials 7 (2006) 14.

[6] X. Zong, K. Kim, D. Fang, S. Ran, B.S. Hsiao, B. Chu, Polymer 43 (2002) 4403.

[7] P.W. Gibson, H.L. Schreuder-Gibson, D. Rivin, AIChE J. 45 (1999) 190.

[8] E.R. Kenawy, Y.R. Abdel-Fattah, Macromol. Biosci. 2 (2002) 261.

[9] C. Burger, B.S. Hsiao, B. Chu, Annu. Rev. Mater. Res. 36 (2006) 333.

[10] J.R. Kim, S.W. Choi, S.M. Jo, W.S. Lee, B.C. Kim, Electrochim. Acta 50 (2004) 69.

[11] X.Y. Wang, C. Drew, S.H. Lee, K.J. Senecal, J. Kumar, L.A. Sarnuelson, Nano Lett. 2 (2002) 1273.

[12] H.Q. Liu, J. Kameoka, D.A. Czaplewski, H.G. Craighead, Nano Lett. 4 (2004) 671.

[13] X.Y. Wang, Y.G. Kim, C. Drew, B.C. Ku, J. Kumar, L.A. Samuelson, Nano Lett. 4 (2004) 331.

[14] B. Ding, J. Kim, Y. Miyazaki, S. Shiratori, Sens. Actuators B 101 (2004) 373.

[15] US Patent, 6,110,588 (2000).

[16] US Patent, 5,935,883 (1999)

[17] US Patent, 6,382,526 (2002).

[18] A.A. Tseng, A. Notargiacomo, T.P. Chen, J. Vac. Sci. Technol. B 23 (2005) 877.

[19] J.C. Huie, Smart Mater. Struct. 12 (2003) 264.

[20] K.M. Woo, V.J. Chen, P.X. Ma, J. Biomed. Mater. Res. A 67A (2003) 531
[21] R. Murugan, S. Ramakrishna, Tissue Eng. 13 (2007) 1845.

[22] A. Martins, J.V. Araújo, R.L. Reis, N.M. Neves, Nanomedicine 2 (2007) 929.

[23] J. Nam, Y. Huang, S. Agarwal, J. Lannutti, Tissue Eng. 13 (2007) 2249.

[24] G. Kim, W. Kim, J. Biomed. Mater. Res. B 81B (2007) 104.

[25] D. Bäuerle, Laser Processing and Chemistry, 3rd ed., Springer-Verlag, Berlin, 2000.

[26] A.A. Serafetinides, M.I. Makropoulo, C.D. Skordoulis, A.K. Kar, Appl. Surf. Sci. 180 (2001) 42.

[27] S. Baudach, J. Bonse, J. Kruger, W. Kautek, Appl. Surf. Sci. 154 (2000) 555.

[28] H. Kweon, M. Yoo, I.K. Park, T.H. Kim, H.C. Lee, H.S. Lee, J.S. Oh, T. Akaike, C.S. Cho, Biomaterials 24 (2003) 801.

[29] E. Piskin, Biodegradable polymers in medicine, in: G. Scott (Ed.), Degradable Polymers: Principles and Applications, Kluwer, Netherlands, 2002, pp. 321-379.

[30] A. Martins, E.D. Pinho, S. Faria, I. Pashkuleva, A.P. Marques, R.L. Reis, N.M. Neves, Small 5 (2009) 1195.

[31] M.H. Hong, S.M. Huang, W.J. Wang, K.S. Tiaw, S.E. Teoh, B. Luk'yanchuk, T.C. Chong, Mater. Res. Soc. Symp. Proc. 780 (2003) 47.

[32] H.W. Choi, J.K. Johnson, J. Nam, D.F. Farson, J. Lannutti, J. Laser Appl. 19 (2007) 225.

[33] F.L. Pendroitti, L.S. Pendroitti, Introduction to Optics, 2nd ed., Prentice Hall, Englewood Cliffs, New Jersey, 1991.

[34] P. Taddei, R. Simoni, G. Fini, J. Mol. Struct. 565-566 (2001) 317.

[35] J.S. Fletcher, X.A. Conlan, N.P. Lockyer, J.C. Vickerman, Appl. Surf. Sci. 252 (2006) 6513. 